\title{
Case Report \\ An accidental finding of FAHR's syndrome during computed tomography of skull - A case report
}

\author{
Archana Rajasundaram ${ }^{1}$, Nitya Waghray ${ }^{2}$, W.M.S. Johnson ${ }^{3}$ \\ ${ }^{1}$ Associate Professor, ${ }^{2} \mathrm{PhD}$ Scholar, ${ }^{3}$ Dean, Professor and HOD, Department of Anatomy, Sree Balaji Medical \\ College and Hospital, BIHER, Chennai, Tamil Nadu
}

(Received: July 2020

Revised: October 2020

Accepted: November 2020)

Corresponding author: Archana Rajasundaram. Email: archana.r@bharathuniv.ac.in

\begin{abstract}
Calcification of basal ganglia is known as FAHR's syndrome or FAHR's disease. It's a rare phenomenon which generally affects the young to middle aged adults. A 39-year-old female was sent to the radiology department of Sree Balaji Medical College, Chennai for computed tomography (CT) as she had a fall and injured her forehead. An axial plain CT scan without contrast was advised and performed. The CT study revealed mild frontal peri cranial swelling and calcification of dentate nucleus, basal ganglia, central semiovale and frontoparietal subcortical white matter was observed. On examination, however patient did not present with any other movement disorders.
\end{abstract}

Keywords: FAHR's Syndrome; calcification; basal ganglia; dentate nucleus

\section{INTRODUCTION}

$\mathrm{F}$ AHR's disease or FAHR's syndrome is a rare, neurological disorder characterized by abnormal calcified deposits in various areas of brain. These deposits which are calcified are made up mainly of calcium carbonate and calcium phosphate. They may be found in the cerebral cortex, thalamus, hippocampus, cerebellar cortical white matter and dentate nucleus. However, they are very commonly seen in basal ganglia either unilaterally or bilaterally.

It was first described by a German neurologist, Karl Theodor Fahr in the year 1930. The clinical features of the disease presents neuropsychiatric, extrapyramidal and cerebellar symptoms, convulsive seizures, parkinsonian features, dementia and speech disorders. However asymptomatic cases have also been reported. The disease usually occurs between 40-60 years of age $(1,2)$.

Molecular genetics of this disease haven't been studied extensively; hence evidence at the molecular and genetic level is limited. FAHR's disease commonly affects middle aged adults. This condition presents with a number of conditions which include

\section{CASE REPORT}

endocrine disorders, dermatological myopathies and several infectious diseases. Clinical manifestations of this disease incorporate a wide variety of symptoms, like neurological symptoms of extrapyramidal movement disorders such as chorea. Bilateral calcification of basal ganglia, progressive neurological dysfunction, absence of an infectious, traumatic or toxic cause and a notable family history are some of the diagnostic criteria for FAHR's disease which have been formulated based on the modifications from previous evidences. Other methods of diagnosis are various imaging modalities like CT, MRI and plain X ray of skull. Other investigations include blood and urine testing for hematologic and biochemical indices. Disease is as yet incurable but management and treatment strategies mainly focus on symptomatic relief; however certain evidence is present to suggest that early diagnosis and treatment can reverse the calcification process leading to complete recovery of mental functions. Families with a known history of FAHR's disease should be counselled prior to conception so that the birth of affected babies can be prevented (3). 

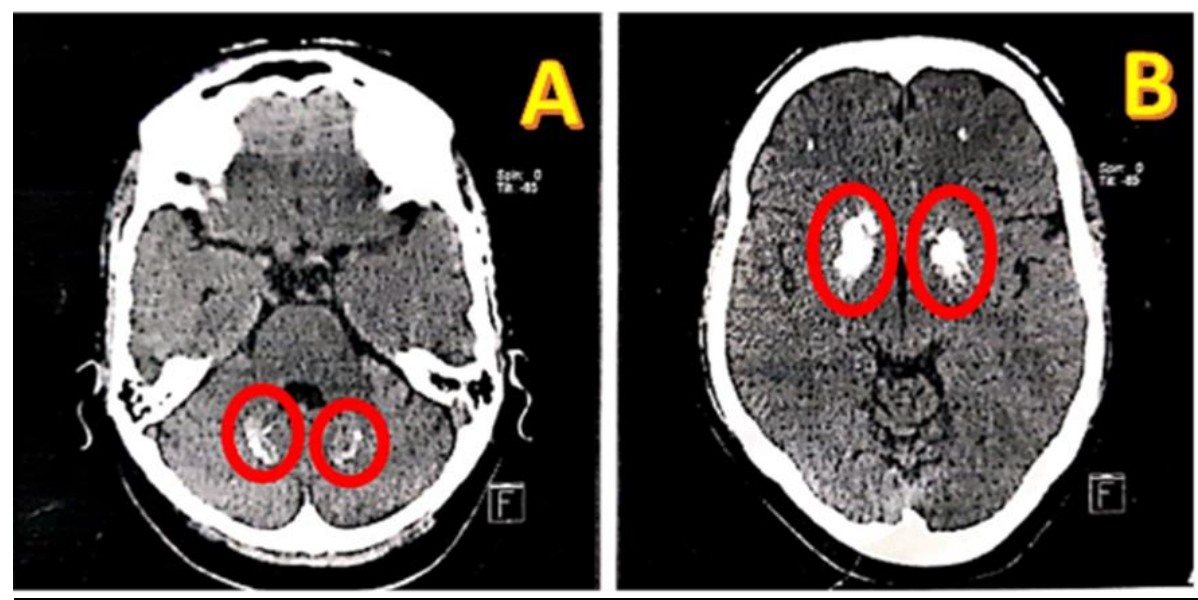

Fig. 1: F- Frontal View CT Scan of a 39-year-old female showing calcifications [A] of dentate nucleus [B] in basal ganglia A 39 years' old female was referred to the radiology department of Sree Balaji Medical College and Hospital, Chennai for CT scan after having a fall and being injured on the frontal surface of the head There was no history of any neuropsychiatric manifestations, mood disturbances or seizures noted. She was moderately built and nourished without any skeletal abnormalities or neurocutaneous markers. She was conscious and was oriented to the time and place, with a normal memory and intelligence. She did not present with any motor weakness or cranial nerve involvement. There was no rigidity, tremor or abnormal movements. Her coordination was intact. Her other systemic examinations were normal. The serum levels of calcium, magnesium, phosphorus and alkaline phosphatase were normal. The assays done for estimating the parathormone and serum vitamin D were found to be normal. CT scan of the brain revealed bilateral areas of calcification over the basal ganglia, dentate nucleus, centrum semiovale, fronto- parietal subcortical white matter which were suggestive of FAHR's disease.

\section{DISCUSSION}

Idiopathic calcification of basal ganglia also known as Bilateral Striopallido Dentate Calcinosis (BSPDC), is a neurodegenerative disorder and is commonly known as FAHR's disease. A bilateral, symmetrical, intra cranial calcification characterizes FAHR's disease with a predilection for the basal ganglia and the dentate nuclei. Because of the symmetrical involvement of these nuclei, the descriptive terminology, BSPDC, has been put forth (3).

This is a very rare disease of unknown prevalence. The typical age at the onset of the symptoms is 40-60 years. As per the FAHR's Disease Registry, this disease most commonly presents with movement disorders and these account for a total of $55 \%$. Out of these $55 \%$, parkinsonism accounts for $57 \%$, chorea was noted in $19 \%$ of the cases, tremor in $8 \%$, dystonia in $8 \%$, athetosis in 5\% and orofacial dyskinesia in $3 \%$ of the cases. The other neurological manifestations include a cognitive impairment, cerebellar signs, speech disorders, pyramidal signs, psychiatric features, gait disorders and sensory changes. The clinical diagnosis

of FAHR's disease is based on the combination of clinical features, brain imaging and on an exclusion of other causes of the intracranial calcification (4). The imaging findings of the symmetric and extensive calcification are usually typical, as was seen in our case. The disorders of calcium metabolism may occur in association with the intracerebral calcification. Intracerebral calcification could be a result of hypoparathyroidism, pseudohypoparathyroidism and hyperparathyroidism. It has been observed that the inheritance has been an autosomal dominant in the familial cases as per some of the genetic studies. A study carried out on a multigenerational family showed the linkage to the IBGC1 of chromosome 14. However, the casual gene still remains unknown.

The Positron emission tomography studies of brain metabolism conducted by Benke et al., in a patient with FAHR's disease presented with the predominant frontal lobe syndrome and dementia. (5). There was a massive reduction of the glucose metabolism in both the basal ganglia and the frontal lobes, which included the orbitofrontal and the anterior cingulate areas, which correlated with the clinical picture of disinhibition and a personality change (6).

Computed tomography scan remains the most effective screening tool. No prenatal or genetic tests are available for genetic counselling. The minimum age at which a negative CT scan can suggest the exclusion of the disease has not been established as yet. To clarify whether the disease is sporadic or familial, doing the imaging scan of the parents and other kindred is more reliable than their clinical screening (7). FAHR's disease is most commonly transmitted as an Autosomal Dominant trait; but it may also be passed on as an autosomal recessive trait or it may occur sporadically $(1,7)$. A Locus at $14 \mathrm{q}$ (IBGC1) has been suggested to be involved commonly. A second locus has been identified on chromosome 8 and a third on chromosome 2 (3).

Endocrine disorders, particularly parathyroid disturbances are most commonly associated with FAHR's syndrome. These abnormalities include idiopathic hypoparathyroidism, secondary hypoparathyroidism, pseudo-hypoparathyroidism, 
pseudo- pseudohypoparathyroidism and hyperparathyroidism. Vitamin D is a known player in the process of calcium metabolism due to which a disturbance in its homeostasis has significant implications for patients with FAHR's syndrome (4).

\section{CONCLUSION}

Basal ganglia calcification is also known as FAHR's disease or FAHR's syndrome. It has a prevalence of $<1 / 1,000,000$ and it is a rare inherited or most cases present with extra pyramidal symptoms initially. Additionally, they may present with cerebellar dysfunction, speech difficulty, dementia and neuropsychiatric symptoms. Idiopathic basal ganglia calcification or FAHR's syndrome is a rare neurological disorder that is passed on in families as an autosomal dominant trait. This disorder is associated with a variety of other diseases but no specific etiologic agent has been identified yet. New treatment modalities need to be discovered and employed to minimize loss of functionality associated with the disease. Of even more importance is to emphasize on the significance of genetic counselling of known at risk parents before conception. Screening asymptomatic individuals has not been able to demonstrate immediate medical benefits in adults or young individuals. It is of significant use only in adults as it can help them in decision making in their personal life regarding matters of finance, marriage and career. Screening of young individuals is considered unnecessary as it can result in psychological harm without being medically beneficial.

\section{CONFLICT OF INTEREST}

The authors declare no conflict of interest.

\section{REFERENCES}

1. Murat, G., Ali, F., Serdar, K., Hasan, B. Fahr's Syndrome Three cases presenting with psychiatric signs. Eur J Gen Med. 2006; 3(1): 35- 40.

2. Kotan, D., Aygul, R. Familial Fahr's disease in a Turkish family. South Med J. 2009; 102(1): 85-86.

3. Saleem, S., Aslam, H. Fahr's syndrome: literature review of current evidence. Orphanet J of Rare Dis. 2013; 8: 156.

4. Billard, C., Dulac, O., Bouloche, J., Echenne, B., Lebon, P., Motte, J. Encephalopathy withcalcifications of the basal ganglia in children. A reappraisal of Fahr's syndrome with respect to 14 new cases. Neuropediatrics. 1989; 20(1): 12-19.

5. Benke, T., Karner, E., Seppi, K., Delazer, M., Marksteiner, J., Donnemiller, E. Subacute dementia and imaging correlates in a case of Fahr's disease. J Neurol Neurosurg Psychiatry. 2004; 75(8): 1163-1165.

6. Geschwind, D. H., Loginov, M., Stern, J. M. Identification of a locus on chromosome 14q. for idiopathic basal ganglia calcification (Fahr's disease) Am J Hum Genet. 1999; 65(3): 764-772.

7. Lam, J. S., Fong, S. Y., Yiu, G. C., Wing, Y. K. Fahr's disease: a differential diagnosis of frontal lobe syndrome. Hong Kong Med J. 2007; 13(1): 75-77. 